\title{
Introducing orthogonal roller pairs as an effective isolating system for low rise buildings
}

\author{
M. Hosseini ${ }^{1} \&$ K. Kangarloo ${ }^{2}$ \\ ${ }^{I}$ The International Institute of Earthquake Engineering and Seismology \\ (IIEES), Tehran, Iran \\ ${ }^{2}$ Graduate School, South Tehran Branch of the Islamic Azad University \\ (IAU), and Member of Young Researchers Club, Tehran, Iran
}

\begin{abstract}
In this paper a new isolating system is introduced which does not need sophisticated manufacturing techniques, and is not costly as other existing systems like lead-rubber bearing, or friction pendulum bearing systems. The proposed system consists of two pairs of orthogonal steel rollers, making possible the movement of the superstructure in all horizontal directions. Rollers move on a cylindrical steel bed, which gives a restoring capability to the system. The two rollers are connected together at both ends with two hinged plates. This makes the two rollers move together and have the same elevation in the cylindrical bed at any instant during the earthquake. The natural period of the system is almost independent of the superstructure mass, and is basically a function of $r / R$ ratio in which $r$ is the radius of the rollers and $R$ is the radius of the cylindrical beds. To obtain the appropriate values of $r$ and $R$ to reach a specific value of the natural period of the isolated system, in addition to analytical hand calculations, some numerical Finite Element calculations have been performed. The calculations have been verified by laboratory tests. Results show that if the rollers and cylindrical beds are made of high-strength steel (MO40 alloy steel) the system can be used effectively buildings up to five stories.
\end{abstract}

Keywords: orthogonal rollers, base isolation, rolling and slope resistance, Hertz contact theory, transfer reaction, contact friction. 


\section{Introduction}

Several isolating systems have been introduced for buildings so far, however, almost all of them need high technology on the one hand, which is not available in many of developing countries, or are still costly for those countries which have the required technology, and on the other hand, the isolators need to be replaced in the case of occurrence of a strong earthquake. Among various types of isolator, rollers have been shown to be to most usable ones, however, some difficulties like distortion of rollers and/or high rolling resistance under extensive loads have hindered their use. Accordingly, although the first applicable cases of these isolators have reported in the late 1980s, (Pham [1], Zastrow [2]), the studies on this type of isolators are ongoing (Tsai et al. [3]).

The idea of "two sets of mutually orthogonal free rolling rods under the basement of the structure" was proposed and tested in early- to mid 90s, (Lin et al. [4], Lin and Hone [5]), however, those cases were limited to light structures or single-story small models. Lin and his colleagues have added a soft spring to the basement to give a re-centering force to the rolling system and reduce the permanent displacement. A roller type isolation system has been also introduced for the individual showcases and individual works of art (Ueda et al. [6]). Their isolation system consists of two layers that form a XY-motion mechanism; each layer consists of wheels, and rails having a circular-linear-combined shape in the vertical cross-section to produce a restoring force. Ueda and his colleague have carried out some shake table tests were, showing good isolation performance of system. Although their system has shown high efficiency, because of using rails and wheels its load bearing capacity is low, and the main usage of their isolating system is in museums objects. Recently, another type of roller isolation system has been proposed by Uematsu et al. [7]. They have claimed that their system can be made of readily available materials and can be easily installed without heavy construction equipment or special skills, however, their system is again basically for light weight structures, and using it for buildings needs some modifications.

It is seen that although various roller isolating systems have been introduced, their capability for usage under high vertical loads in the range of vertical forces of multistory buildings columns is not so much developed yet. In this paper a somehow new isolating system is introduced which is suitable for using in multistory buildings up to five stories. The specifications of this system are explained in the next section of the paper.

\section{The proposed isolating system}

The proposed system basically consists of two pairs of orthogonal steel rolling rods, which can move on separate cylindrical steel beds as shown in fig. 1 .

It can be seen in fig. 1 that because of orthogonal setting of rollers pairs the top plate can move in all horizontal directions. The concave beds give a restoring capability to the system. The two rollers of each pair are connected together at both ends with two plates with hinge connections as shown in fig. 2 . 


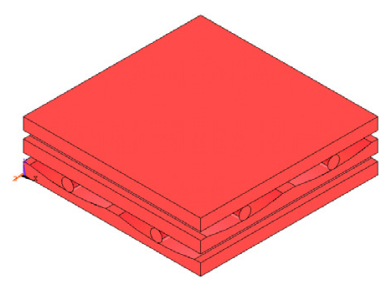

Figure 1: The proposed base isolation system with orthogonal rollers.
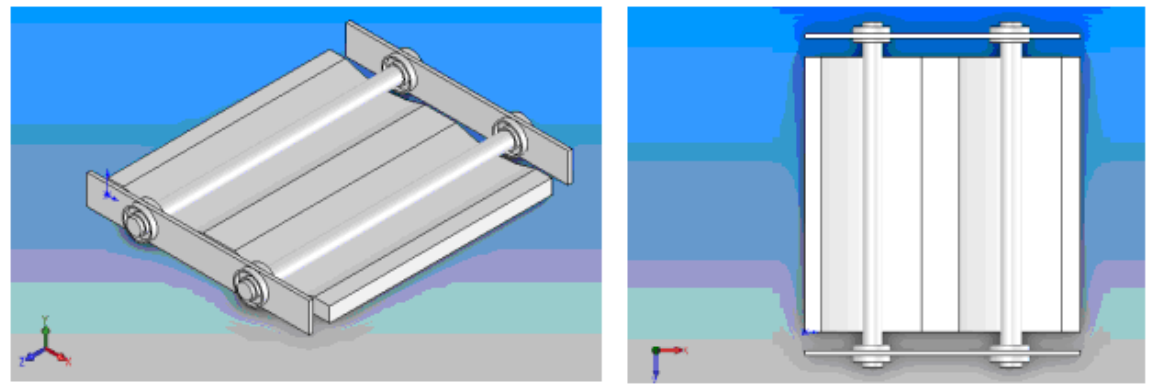

Figure 2: $\quad$ Plates connecting rollers for synchronized motion.

This makes the two rollers of each pair to move together and have the same elevation in the cylindrical beds at any instant during the earthquake, otherwise they may lose their parallel status because of seismic disturbances, particularly the vertical excitations of ground motions.

\section{The system equation of motion}

To drive the equation of motion for the isolated system, it is assumed that the superstructure moves on the isolating system as a rigid body with total mass of $\mathrm{M}$ as shown in fig. 3.

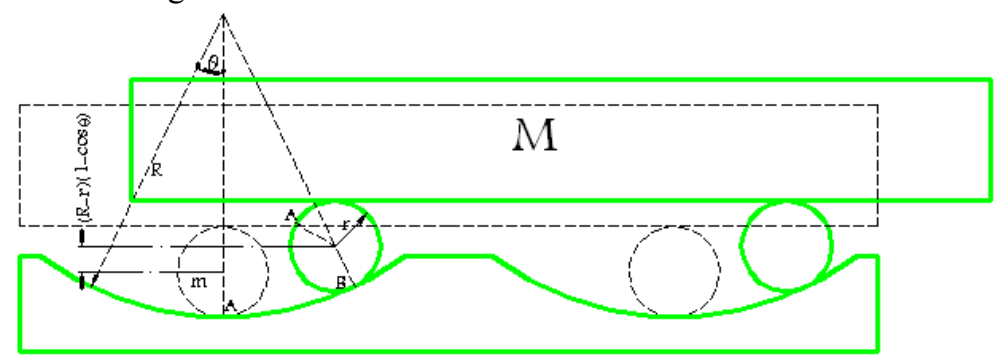

Figure 3: The rigid body model of the superstructure in the isolator.

The mass of rollers is negligible in comparison with M. If $\mu=\operatorname{tg} \theta$ ( $\mu$ is coefficient of sliding friction and $\theta$ is the generalized coordinate for motion of 
the system as shown in fig. 3), the rollers simply roll between surfaces without slippage. On this basis by using LaGrange equation, $\frac{d}{d}\left(\frac{\partial T}{\partial \dot{\theta}}\right)-\frac{\partial T}{\partial \theta}+\frac{\partial V}{\partial \theta}=Q_{\theta}$, the differential equation of motion is obtained as:

$$
\ddot{x}+\frac{g}{(R-r)} x=-\operatorname{sgn}(\dot{x}) \frac{b}{r} g-\ddot{u}_{g}
$$

In eqn (1) $x$ is the relative lateral displacement of the superstructure, $R$ and $r$ are respectively radius of bed and rollers, $b$ is the coefficient of rolling resistan, explained in section $3, g$ is the gravity factor, and $\ddot{u}_{g}$ is the ground acceleration. The period of the system, $T_{n}$, and its restoring force, $p$, are given by:

$$
T_{n}=4 \pi \sqrt{\frac{(R-r)}{g}} \quad, \quad p=\frac{M g}{(R-r)}
$$

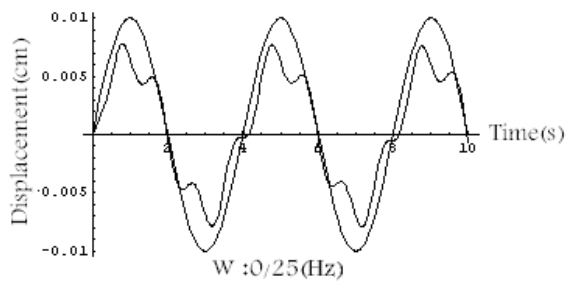

A : $1(\mathrm{~cm})$

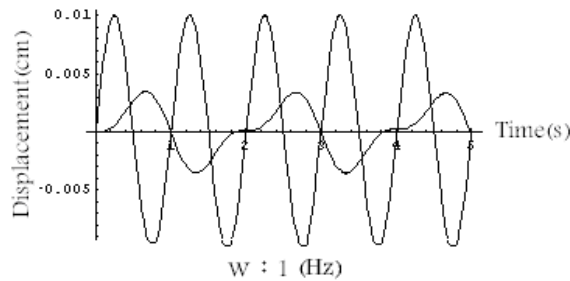

A : $1(\mathrm{~cm})$

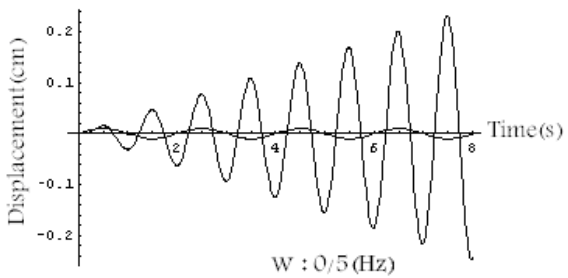

A $: 1(\mathrm{~cm})$

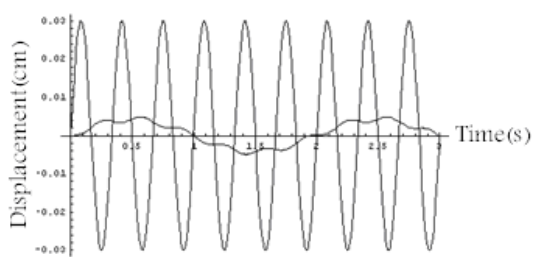

$\mathrm{W}: 3(\mathrm{~Hz})$

A $: 3(\mathrm{~cm})$

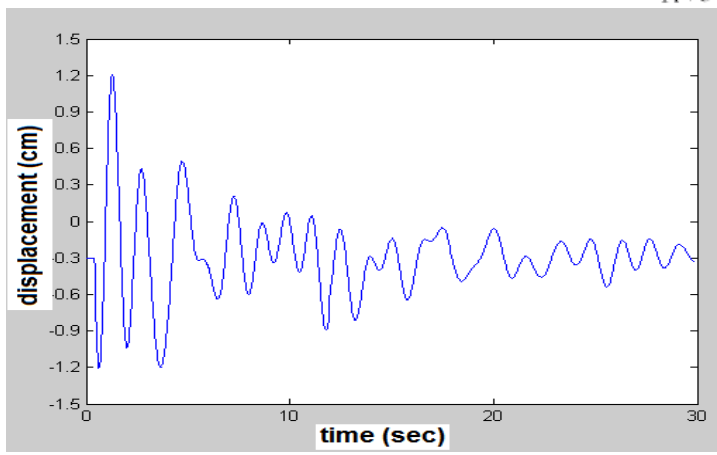

Figure 4: Responses of the system to harmonic and earthquake loadings. 
As it is seen, the natural period of the system is independent of the superstructure mass. The main affecting parameters in the value of natural period of the isolated system are the radii of curvatures of rollers and bed. By assuming $r=2 \mathrm{~cm}$ and $R=26 \mathrm{~cm}$, and solving the equation of motion for the harmonic force of $A \sin (\omega t)$, and also El Centro earthquake accelerogram (reduce to $50 \%$ ) of the obtained responses are as shown in fig. 4 .

It is seen that for $\omega=0.5 \mathrm{~Hz}$ the resonance response has occurred, since the period of the system, given by eqn (2) is 2.0 seconds in this case.

\section{Rolling resistance}

The major source of resistance during rolling motion is the deformations that occur when the two surfaces are compressed together. At the contact points, the roller flattens, while a small trench is formed in the surface (see fig. 5).

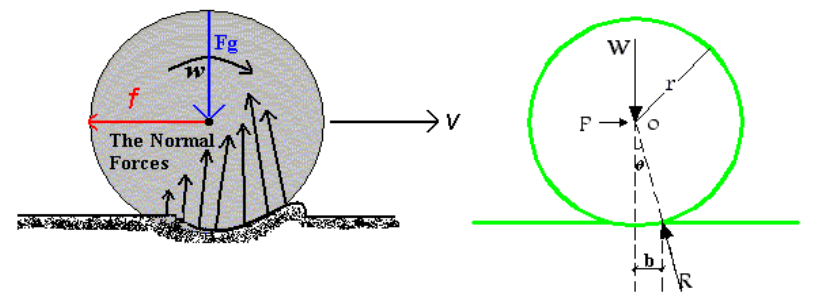

Figure 5: The actual forces acting between the rolling rod and the surface.

The overall rolling resistance or friction results in a force at the center of the wheel and is parallel to the surface of contact, and is represented eqns (3).

$$
P . r=W . b \quad, \quad M_{r}=W . b . r
$$

In eqn (3) $b$ is called Rolling Resistance Parameter. It has the physical dimension of length and its value depends to several factors such as rotating speed, applied pressure or force, roughness of the surfaces, etc. that are not represented clearly. The value of $b$ varies from $25 \mathrm{~mm}$ for steel wheel on the steel rail up to $125 \mathrm{~mm}$ or higher for the steel wheel on the ground.

\section{Lateral stiffness of the system}

Seismic design by using the Design Spectra needs two main parameters, the natural period and the damping coefficient. In the case of rolling systems these parameters are directly related to the lateral stiffness of roller bearings as:

$$
T=2 \pi \sqrt{\frac{M}{K}} \quad, \quad \xi=E /\left(4 \pi \frac{K D^{2}}{2}\right)
$$

where $E$ is the energy dissipated by the bearing system in one sinusoidal cycle, $D$ is the maximum lateral displacement, and $K$ is the stiffness of the system $(K=F / D)$. Force-displacement behavior of the roller bearing is shown in fig. 6 . 
156 Earthquake Resistant Engineering Structures VI

(a) Force due to slope

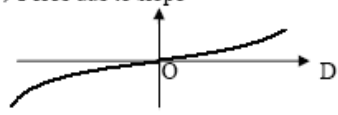

(b) Force due to friction

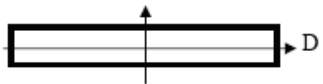

(c) Total force

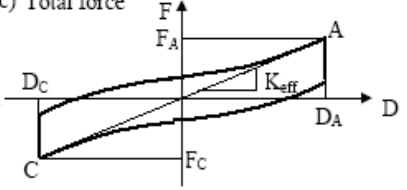

$\mathrm{K}_{\mathrm{eff}}=\left(\mathrm{F}_{\mathrm{A}}-\mathrm{F}_{\mathrm{C}}\right) /\left(\mathrm{D}_{\mathrm{A}}-\mathrm{D}_{\mathrm{C}}\right)$

Figure 6: $\quad$ Force-displacement behavior of the roller bearing.
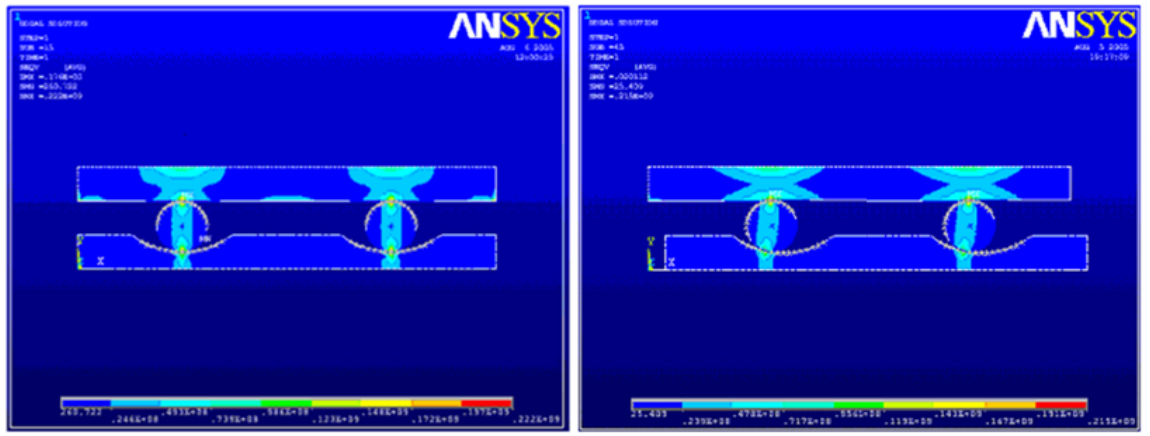

Figure 7: Von Misses stresses for the case of $1.0 \mathrm{~cm}$ lateral movement.
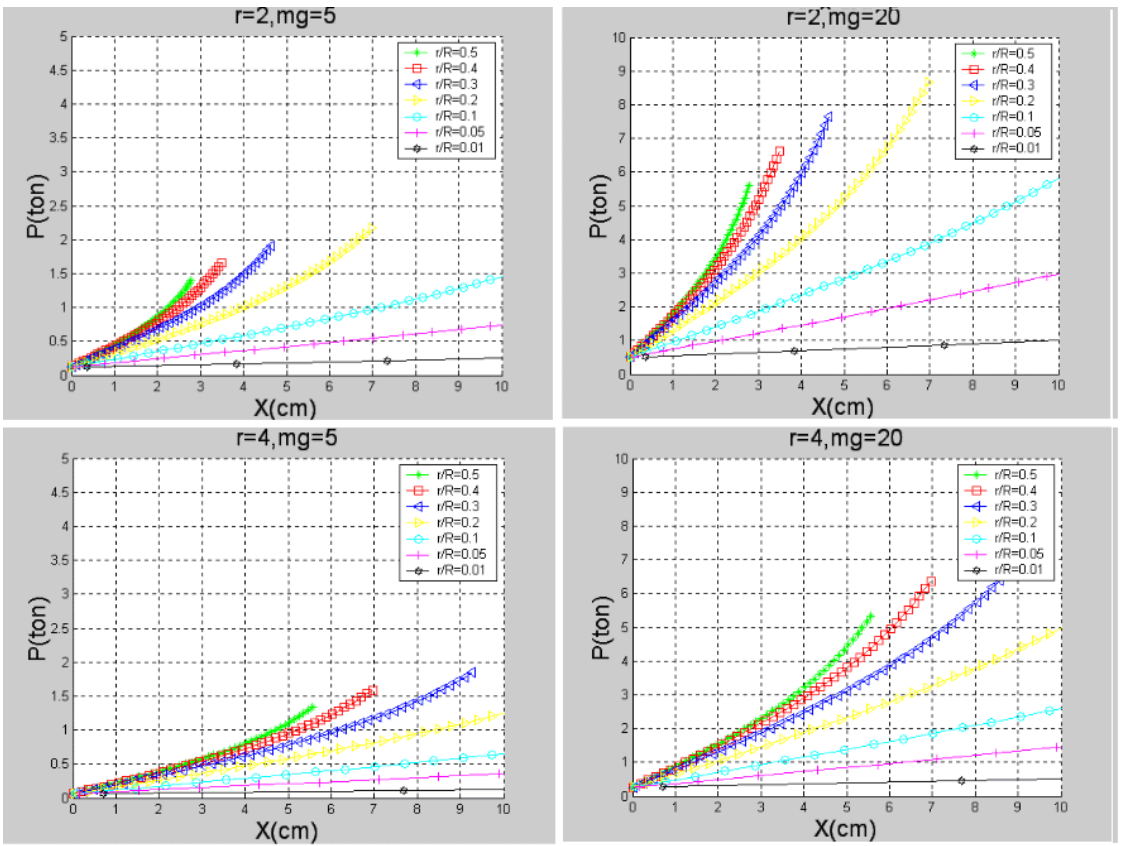

Figure 8: $\quad$ Force-displacement graphs of the system for different ratio of $\mathrm{r} / \mathrm{R}$. 
For analytical stuffy of the lateral stiffness of the system, ANSYS program has been used making benefit from its large displacement option. Fig 7 shows the Von Misses stress values for the case of $1.0 \mathrm{~cm}$ lateral movement. The forcedisplacement graphs, obtained by analyses for different $r / R$ values, varying from 0.01 to 0.5 , using two values of $2 \mathrm{~cm}$ and $4 \mathrm{~cm}$ for $r$, and two values of $5.0 \mathrm{kgf}$ and $20.0 \mathrm{kgf}$ for the vertical load on the rollers are shown in fig. 8 .

It is seen in fig. 8 that in all cases as the $r / R$ value increases the system tends to nonlinear behavior, and shows a hardening state. The effect of higher vertical load on the lateral stiffness of the system is also observed in fig. 8, which is in good agreement with the calculated values obtained by eqn (4).

\section{The vertical stiffness}

When two elastic surfaces are pushed together a contact area is created with high stress in materials, for example, the settlement of a cylinder over another cylinder or a sphere over another sphere. These cases can be described by Hertz contact theory [9]. For two cylinders under a force F, as shown in fig. 9, the length of contact area and the peak pressure can be calculated by the following eqns:

$$
a=\sqrt{\frac{2 \mathrm{~F}\left(1-v^{2}\right)\left[\frac{1}{\mathrm{E}_{1}}+\frac{1}{\mathrm{E}_{2}}\right]}{\pi \cdot \mathrm{L}\left[\frac{1}{\mathrm{~d}_{1}}+\frac{1}{\mathrm{~d}_{2}}\right]}}, \quad \mathrm{P}_{\max }=\frac{2 \mathrm{~F}}{\pi \cdot \mathrm{a} \cdot \mathrm{L}}
$$
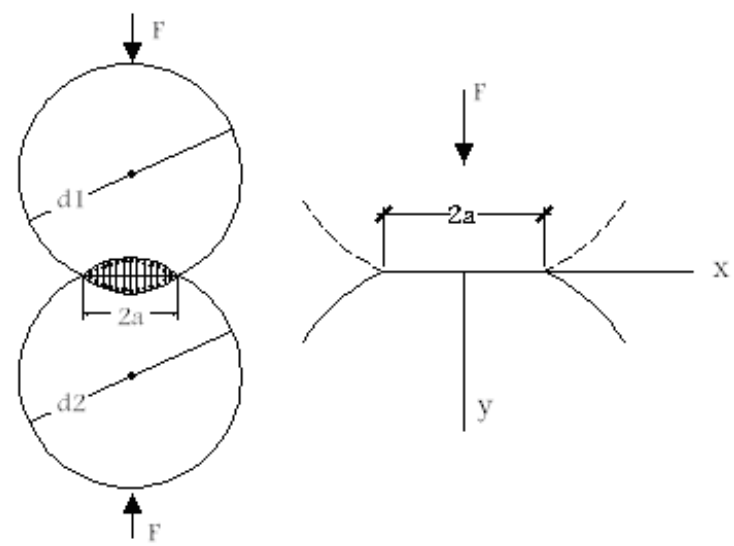

Figure 9: Hertz contact theory for two cylinders. 
In eqns (5) $v$ is the Poison ratio, and $E_{1}$ and $E_{2}$ are respectively the modulus of elasticity of the two materials, and $\mathrm{L}$ is the length of cylinders. In the case of a cylinder on a flat surface the radius of the flat surface is considered as $\infty$ in the formula, and in the case of a cylinder on the internal surface of another bigger cylinder, the radius of the bigger cylinder is used with the negative sign. In the latter case the max shear stress, created in the contact area is about $30 \%$ of the maximum normal stress, namely $0.3 \sigma_{z, \max }$, and occurs around $0.39 \mathrm{a}$ below the surface [9].

To find out the accuracy of finite element analyses a simple problem based on the above discussion has been solved by ANSYS program. Fig. 10 shows the results of this analysis in comparison with the analytical values obtained by Hertz contact theory [9]. As it is seen in fig. 10 there is a good agreement between numerical and analytical results. Of particular interest may be the maximum shear stress and its location which can be seen in the fig. clearly. Based on the good agreement between numerical and analytical results the calculations performed by using ANSYS program are verified, and the numerical results for other similar cases such as the case for calculating the lateral stiffness can be trusted. Accordingly, it is expected that the tests results shows good agreement with numerical results as well.
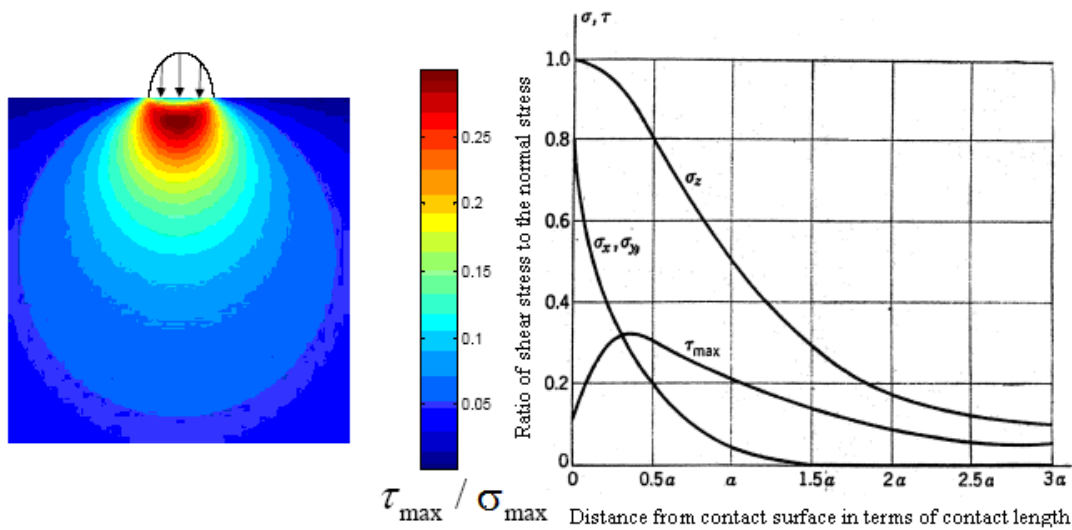

Figure 10: Comparing the numerical and analytical results of the contact problem.

\section{The laboratory tests}

For studying the performance of the proposed system in different directions and to find out the effect of superstructure's weight on the system performance, some shaking table and cyclic or pseudo-dynamic tests have been carried out. At first, 
by using a rigid body model, as shown if fig. 11, behavior of the isolating system subjected to lateral excitations were studied.

Fig. 12 represents the response histories of the system in two orthogonal directions to the a series of harmonic excitations with frequencies of $0.25,0.5,1$, 3 , and $5 \mathrm{~Hz}$, and also to the El Centro earthquake all applied to the system at an angle of 45 degrees with respect to the main axes of the rollers. The masses used in this test were $50 \mathrm{~kg}, 110 \mathrm{~kg}$, and $400 \mathrm{~kg}$, but no difference was observed in the results, as expected. It is seen in fig. 12 that in no case the displacement value has exceeded $5.0 \mathrm{~cm}$, and in the case of earthquake the values are less than 1.0 $\mathrm{cm}$. It can also be seen that the maximum displacements in two orthogonal directions are almost the same, although the time histories are not quite similar in those two directions.

During the first series of tests it was realized that the rollers can not remain parallel during their motion. It can be because of the imperfection in the production process of rollers and their beds, which have been resulted into some undesired motions as they roll in their bed. To overcome this difficulty two thin and narrow plate with hinge connections were added to the system (see fig. 2), and the next test were preformed on the improved system. In the next step of experimental studies, by using actuators, the sample model (made of high strength - MO40 steel alloy) was loaded with different vertical loads and at the same time was pushed and pulled laterally to see how the value of vertical load affect the lateral force-displacement of the system. The observed result, which can not be presented here due to lack of space, were in very good agreement with the numerical results shown in figs 6 and 7. The maximum applied vertical load was $700 \mathrm{kN}$, which in about the column force of a five story ordinary building.

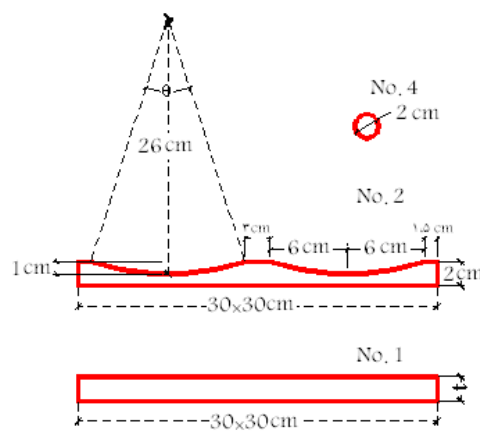

(a)

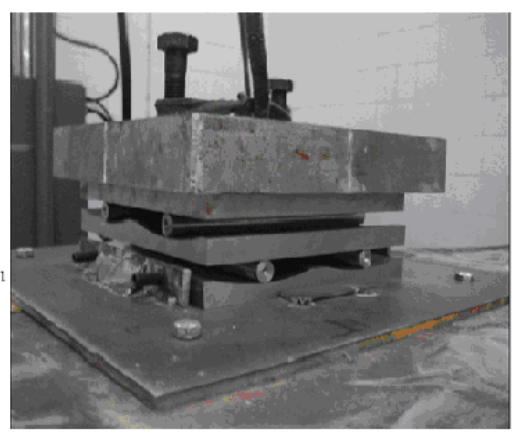

(b)

Figure 11: The fist stage of the laboratory tests by rigid body model. (a) Geometry of the test model, (b) the test model on the shaking table. 
160 Earthquake Resistant Engineering Structures VI
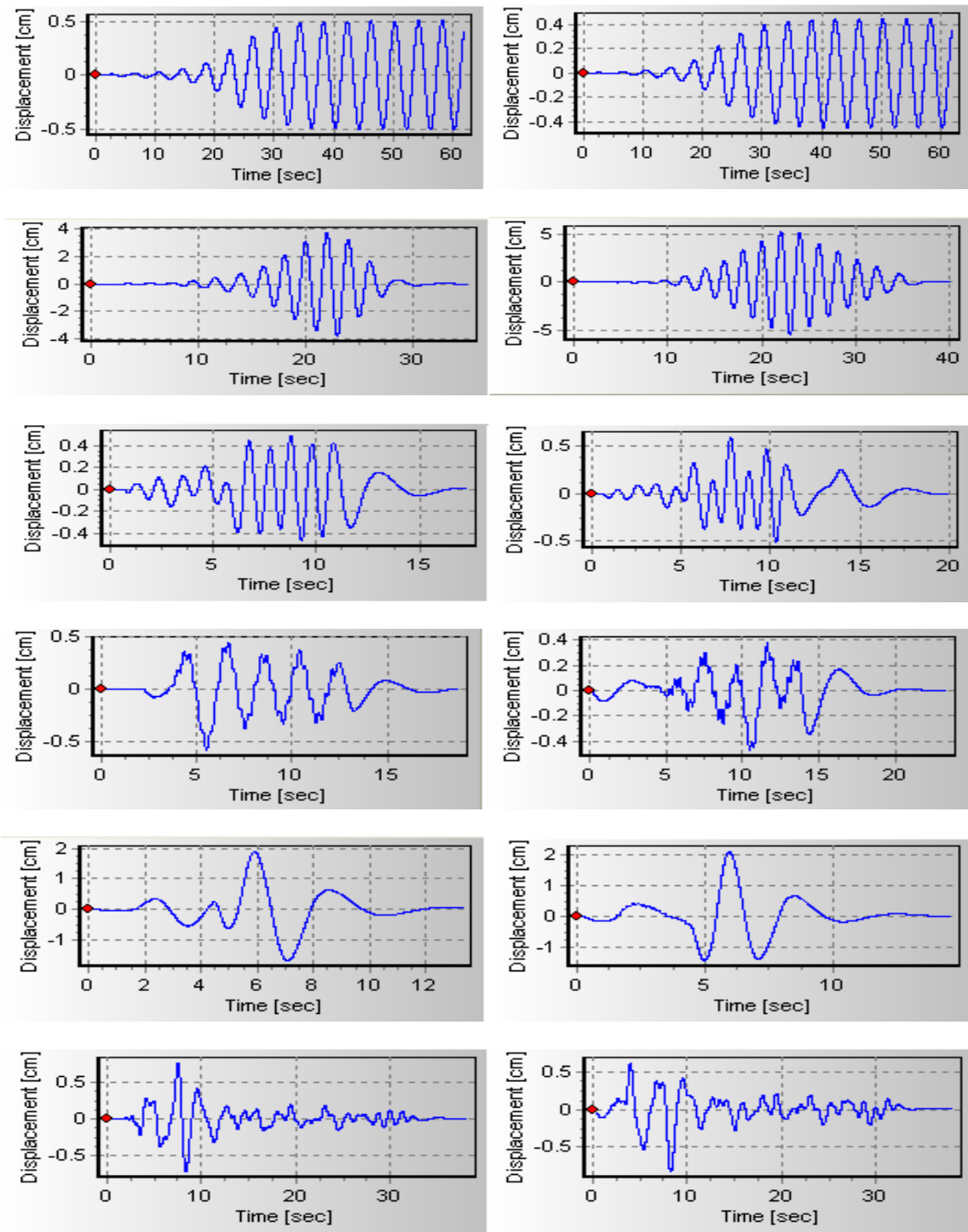

Figure 12: Response histories of the system to harmonic and earthquake loadings.

\section{Conclusions}

Based on the analytical, numerical and experimental results it can be said that:

- The system has good performance subjected to horizontal excitation in every direction. 
- The natural period of the system can be easily controlled by the $r / R$ value, and getting a period of 2.5 second or more in not difficult.

- The maximum lateral displacement of the system under earthquake excitations can be kept limited to a few centimeters by using higher values of $r / R$, provided that the dominant frequency of the earthquake is not low.

Finally, it should be noted that the dimensions of the base isolation systems are important, because there is always a desire for simplicity in installation and low cost. The range of horizontal motion of rollers should be at least equal to the spectral displacement used in the region for seismic design.

\section{References}

[1] Pham, L. T., A base-isolation design using spherically ended rollers and telescopic shock absorbers, Bulletin of the New Zealand National Society for Earthquake Engineering. Vol. 21, no. 2, pp. 135-139, June 1988.

[2] Zastrow, J. B., Rolling with the Big One: base isolation will cushion the jolting, Northern California Real Estate Journal. Vol. 4, no. 4, pp. 5-6. 20, Nov. 1989.

[3] Tsai, Meng-Hao; Chang, Kuo-Chun; and Wu, Sih-Yi, Shaking Table Tests of a Scaled Bridge Model with Rolling-Type Seismic Isolation Bearings, 100th Anniversary Earthquake Conference, 2006.

[4] Lin, T. W., Chern, C. C. and Hone, C. C., Experimental study of base isolation by free rolling rods, Earthquake Engineering \& Structural Dynamics, Vol. 24, no. 12, pp. 1645-1650, Dec. 1995.

[5] Lin, T. W. and Hone, C. C., Base isolation by free rolling rods under basement, Earthquake Engineering \& Structural Dynamics, Vol. 22, no. 3, pp. 261-273, Mar. 1993.

[6] Ueda, Satoshi; Enomoto, Takao; and Fujita, Takafumi, Experiments and Analysis of Roller Type Isolation Device, Proceedings of 13 WCEE, 2004.

[7] Uematsu, Takeyoshi; et al., Development of Compact Vibration Isolation Equipment Applicable to Existing Residences - Restoring Mechanism Utilizing Roller Bearings, Proceedings of 13 WCEE, 2004.

[8] Liang, Z. Song, J. Wang, J. and Lee, G. C., A Sloping Surface Roller Bearing System for Seismic Isolation of Highway Bridges, Technical Report, MCEER, State University of New York at Buffalo, 2003.

[9] Johnson, K. L., Contact Mechanics, Cambridge University Press, 1985. 